\title{
Hepatitis B Vaccination Response in Hemodialysis Patients: The Impact of Dialysis Shift
}

\author{
Maggie Han ${ }^{a}$ Xiaoling Ye ${ }^{a}$ Sharon Rao ${ }^{b}$ Schantel Williams ${ }^{c}$ \\ Stephan Thijssen $^{\text {a Jeffrey Hymes }}{ }^{d}$ Franklin W. Maddux ${ }^{e}$ Peter Kotanko ${ }^{a, c}$ \\ aRenal Research Institute, New York, NY, USA; ${ }^{b}$ School of Medicine, University of Nottingham, Nottingham, UK;

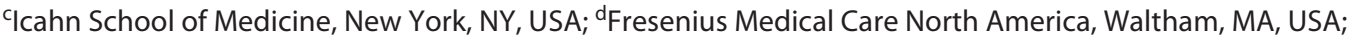 \\ eFresenius Medical Care Management AG, Bad Homburg, Bad Homburg, Germany
}

\section{Keywords}

Hemodialysis · Hepatitis B vaccination · Sleep · Dialysis shift · Seroconversion

\begin{abstract}
Background/Aims: Hepatitis B (HB) vaccination in hemodialysis patients is important as they are at a higher risk of contracting HB. However, hemodialysis patients have a lower HB seroconversion rate than their healthy counterparts. As better sleep has been associated with better seroconversion in healthy populations and early hemodialysis start has been linked to significant sleep-wake disturbances in hemodialysis patients, we examined if hemodialysis treatment start time is associated with HB vaccination response. Methods: Demographics, standard-of-care clinical, laboratory, and treatment parameters, dialysis shift data, $\mathrm{HB}$ antigen status, $\mathrm{HB}$ vaccination status, and $\mathrm{HB}$ titers were collected from hemodialysis patients in Fresenius clinics from January 2010 to December 2015. Patients in our analysis received $90 \%$ of dialysis treatments either before or after 8:30 a.m., were negative for $\mathrm{HB}$ antigen, and received a complete series of $\mathrm{HB}$
\end{abstract}

vaccination (Engerix $\mathrm{B}^{\circledR}$ or Recombivax $\mathrm{HB}^{\mathrm{T}}{ }^{\mathrm{M}}$ ). Univariate and multivariate regression models examined whether dialysis start time is a predictor of $\mathrm{HB}$ vaccination response. Results: Patients were 65 years old, $57 \%$ male, and had a HD vintage of 10 months. Patients whose dialysis treatments started before 8:30 a.m. were more likely to be younger, male, and have a greater dialysis vintage. Patients receiving Engerix $\mathrm{B}^{\circledR}$ and starting dialysis before 8:30 a.m. had a significantly higher seroconversion rate compared to patients who started dialysis after 8:30 a.m. Early dialysis start was a significant predictor of seroconversion in univariate and multivariate regression including male gender, but not in multivariate regression including age, neutrophil-to-lymphocyte ratio, and vintage. Conclusion: While better sleep following vaccination is associated with seroconversion in the general population, this is not the case in hemodialysis patients after multivariate adjustment. In the context of end-stage kidney disease, early dialysis start is not a significant predictor of $\mathrm{HB}$ vaccination response. The association between objectively measured postvaccination sleep duration and seroconversion rate should be investigated.

(c) 2021 S. Karger AG, Basel

$\begin{aligned} & \text { karger@karger.com } \\ & \text { www.karger.com/bpu }\end{aligned}$
Karger ${ }^{\prime /}$

Maggie Han

Research Department, Renal Research Institute

315 East 62nd Street, 4th floor

New York, NY 10065 (USA)

maggie.han@ rriny.com 


\section{Introduction}

Hemodialysis (HD) to sustain life of end-stage kidney disease (ESKD) patients requires access to the patients' blood compartment for prolonged periods of time. As a result, there is an increased risk for transmission (directly or indirectly) of $\mathrm{HB}$ virus in an in-center HD setting where many patients are treated. HB vaccination is therefore recommended for nonprotected HD patients. However, it has been well documented that the ESKD population exhibits lower levels of both innate and adaptive immunity as a result of kidney dysfunction $[1,2]$. The inhibited function of the immune system in the ESKD population demands that vaccination be optimized with respect to number, dosing, and timing of vaccine administrations, to increase seroconversion rates.

The role of sleep in adaptive immunity has been well established in the general population. In healthy populations, chronic inadequate sleep or sleep disturbances following vaccination is associated with diminished immune response and immunologic memory $[3,4]$. In a randomized controlled trial, it was found that following several nights of partial sleep deprivation, response to the influenza vaccination, measured by anti-influenza IgG titers, was diminished over 50\% [5]. Evidence has shown that subjects who reported poor sleep quality were more susceptible to the common cold following exposure to a rhinovirus [6]. Better quality sleep, and specifically more slow-wave sleep which occurs early in the night, has been found to promote human Th1 immune responses, leading to stronger immunological memories [7].

The biological mechanisms behind why sleep influences adaptive immunity have been explored [3, 8]. Greater sleep duration leads to an increase in cellular responses via increased Thl cytokine activity and phagocyte-dependent responses [3]. In addition, a lack of adequate sleep increases systemic inflammation which leads to lower immune response. Adequate sleep also enhances $\mathrm{T}$-cell and B-cell differentiation through IL-2 production, which in turn contributes to antibody production and immune responses [8].

Chronic HD patients experience sleep disturbances during the night due to a plethora of maladies, for example, sleep apnea and restless leg syndrome $[9,10]$. A recent study found that sleep schedules of HD patients are significantly affected by their treatment start time [11]. Compared to patients who receive HD treatments later in the day, patients who are dialyzed early in the morning (starting between 6:00 and 10:00 a.m.) experience altered sleep/wake patterns, that is, they sleep significantly less the night before HD and more the night following HD [11]. Given the immunomodulatory effect of sleep disturbance on vaccination responses documented in other populations, the goal of this study was to investigate whether there is an association between early morning $\mathrm{HD}$ start and $\mathrm{HB}$ vaccination outcomes.

\section{Methods}

\section{Selection Criteria}

In this retrospective cohort study, all the patients ( $\geq 18$ years old) who started their in-center HD with Fresenius Medical Care North America (FMCNA) (i.e., either incident to dialysis or new to FMCNA) from January 2010 to December 2015 were included. Patients were initially screened for the presence of HB surface antigen (HBsAg) before they started their in-center treatments. Patients who tested negative of $\mathrm{HB}$ surface antigen were referred to undergo $\mathrm{HB}$ serology testing to determine the titer of anti-HBsAg antibodies. Patients who tested negative for antibody (titers $<10$ $\mathrm{IU} / \mathrm{L}$ ) were considered susceptible to $\mathrm{HB}$ infection; therefore, they were required to have $\mathrm{HB}$ vaccinations administrated. Two vaccines were used in FMCNA clinics: Engerix B ${ }^{\circledR}$ (SmithKline Beecham Biologicals, Philadelphia, PA, USA) and Recombivax $\mathrm{HB}^{\mathrm{TM}}$ (Merck \& Co, West Point, PA, USA). Per FMCNA's protocol, for patients who received Engerix $B^{\circledR}$, the $2 \mathrm{nd}$, 3rd, and 4 th doses were administrated around 1, 2, and 6 months after the initial dose. For patients who received Recombivax $\mathrm{HB}^{\mathrm{TM}}$, the 2 nd and 3 rd doses were administrated 1 and 5 months after the initial dose. All doses are administered intramuscularly (i.m.) into the deltoid muscle.

\section{Definition of Complete Vaccination Series}

To allow for a reasonable degree of operational deviation and yet arrive at a clean analysis cohort for investigation of vaccination outcomes, we defined a "complete" vaccination series as follows:

Engerix $B^{\circledR}(40 \mu \mathrm{g}$ i.m. each dose):

- Dose 1: day 0

- Dose 2: day $30 \pm 10$

- Dose 3: day $60 \pm 10$

- Dose 4: between dose 3 and day 360

Recombivax $\mathrm{HB}^{\mathrm{TM}}(40 \mu \mathrm{g}$ i.m. each dose):

- Dose 1: day 0

- Dose 2: day $30 \pm 10$

- Dose 3: between dose 2 and day 360

Patients who finished the 1 st complete series of either vaccination were included in the final analysis. We excluded patients who switched vaccines before completing a series of vaccination.

\section{Definition of "Early" versus "Late" Dialysis Shift}

For stratification into "early" versus "late" dialysis shift, we considered patients who started $90 \%$ of their HD treatments (during the 1st completed vaccination series) before 8:30 a.m. as "early," while at 8:30 a.m. or later as "late." The primary reason that we choose a treatment start time earlier than 8:30 a.m. (taking into account time for breakfast, time for brushing teeth, showering, shaving, etc., time for transportation to the clinic, and waiting time in the clinic) would arguably entail a very early rise for 


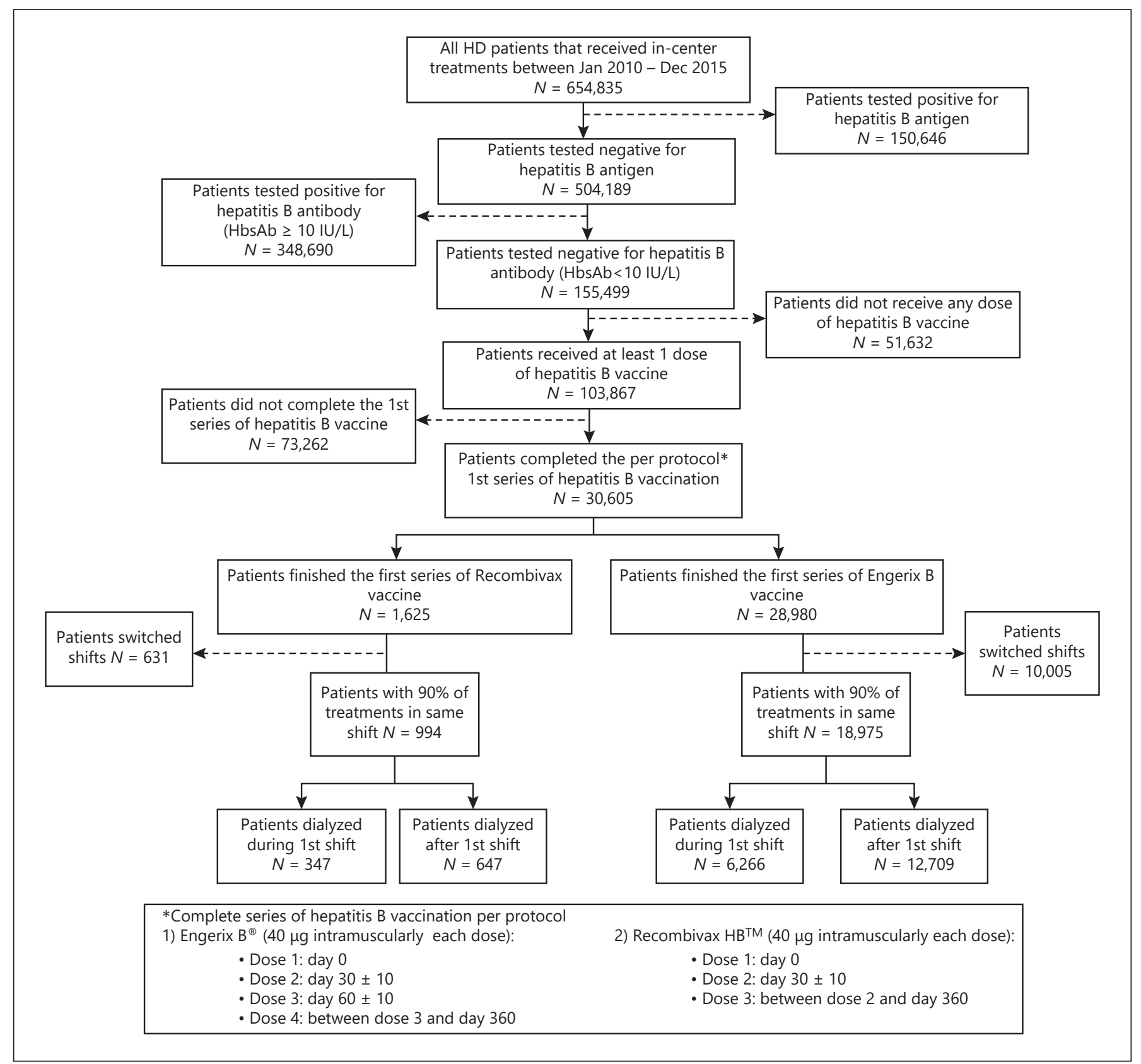

Fig. 1. Flowchart of study cohort. HD, hemodialysis.

the majority of patients by most standards, which in turn could be associated with sleep-wake cycle disturbances. Second, a recent study demonstrated significantly altered sleep-wake patterns in patients who start their HD treatments between 6:00 and 10:00 a.m., which is in line with the assumption of current study [11]. A cutoff of 8:30 a.m. is stricter than the criterion employed by the study referenced above while still maintaining an adequate number of patients in the "early" group to allow for meaningful analyses.

\section{Vaccination Outcomes}

A vaccination was deemed successful, and a patient clinically protected against infection, if an HBsAb titer of $\geq 10$ IU/L was developed after hepatitis $B$ vaccination. The first titer value, up to 1 year following the final vaccination, was used to determine vaccine response. Any titer value of $<10$ IU/L or taken at $<23$ days following the final dose was deemed inconclusive due to inadequate time for antibody production, and the subsequent titer value was taken. Titer values of $\geq 10$ IU/L taken $<23$ days after vaccination were considered successful seroconversion. The 23 days' cutoff was de- 
Table 1. Descriptive statistics of study cohort

\begin{tabular}{|c|c|c|c|c|}
\hline Variable & $\begin{array}{l}\text { Entire cohort } \\
(N=19,969) \\
\text { mean } \pm \text { SD }\end{array}$ & $\begin{array}{l}\text { Before } 8: 30^{*} \\
(n=6,613) \\
\text { mean } \pm \text { SD }\end{array}$ & $\begin{array}{l}\text { After } 8: 30^{*} \\
(n=13,356) \\
\text { mean } \pm \text { SD }\end{array}$ & $p$ value \\
\hline Neutrophil-to-lymphocyte ratio & $4.13 \pm 8.15$ & $3.72 \pm 2.27$ & $4.32 \pm 9.72$ & $<0.0001$ \\
\hline nPCR, g/kg/day & $0.91 \pm 0.31$ & $0.92 \pm 0.37$ & $0.91 \pm 0.28$ & 0.0023 \\
\hline $\mathrm{eKt} / \mathrm{V}$ & $1.55 \pm 0.29$ & $1.56 \pm 0.28$ & $1.55 \pm 0.29$ & 0.1293 \\
\hline $\mathrm{BMI}, \mathrm{kg} / \mathrm{m}^{2}$ & $29.54 \pm 9.69$ & $30.48 \pm 10.28$ & $29.06 \pm 9.34$ & $<0.0001$ \\
\hline Vintage, years & $0.86 \pm 1.61$ & $1.2 \pm 1.83$ & $0.69 \pm 1.46$ & $<0.0001$ \\
\hline Square root of HD vintage & $0.70 \pm 0.63$ & $0.86 \pm 0.70$ & $0.62 \pm 0.57$ & $<0.0001$ \\
\hline $\mathrm{WBC}, 1,000 / \mu \mathrm{L}$ & $7.22 \pm 2.17$ & $7.12 \pm 2.17$ & $7.27 \pm 2.17$ & $<0.0001$ \\
\hline Hemoglobin, g/dL & $11.22 \pm 0.77$ & $11.27 \pm 0.79$ & $11.20 \pm 9.75$ & $<0.0001$ \\
\hline Body surface area, $\mathrm{m}^{2}$ & $1.90 \pm 0.27$ & $1.95 \pm 0.27$ & $1.88 \pm 0.26$ & $<0.0001$ \\
\hline Race: white, $\%$ & 69.42 & 66.84 & 70.69 & $<0.0001$ \\
\hline $\mathrm{CHF}, \%$ & 13.06 & 12.11 & 13.55 & 0.0157 \\
\hline Cancer, other than skin, \% & 2.49 & 2.55 & 2.46 & 0.7463 \\
\hline 3 HD treatments per week, $\%$ & 50.42 & 50.50 & 50.28 & 0.7934 \\
\hline Vaccine type: Engerix B, \% & 95.02 & 94.75 & 95.16 & 0.2178 \\
\hline HBsAb titer of $\geq 10 \mathrm{IU} / \mathrm{mL}, \%$; vaccinated with Engerix B & 72.90 & 74.10 & 72.20 & 0.006 \\
\hline HBsAb titer of $\geq 10 \mathrm{IU} / \mathrm{mL}, \%$; vaccinated with Recombivax $\mathrm{HB}$ & 63.60 & 66.90 & 61.80 & 0.13 \\
\hline
\end{tabular}

nPCR, normalized protein catabolic rate; BMI, body mass index; WBC, white blood cells; CHF, chronic heart failure; eKT/V, dialysis adequacy. $* 90 \%$ of treatment start time during the observation period falls into this category.

rived from the FMCNA policy that titer measurements should be obtained at the earliest 1-2 months postvaccination, and a 1-week window was given for operational variation. Titer measurements were performed by Spectra Renal Laboratory, Rockleigh, NJ, USA.

Demographic, Treatment, Clinical, and Laboratory Parameters

The following demographic and clinical data were collected over the course of the vaccination period: age, race (white vs. nonwhite), gender, vintage (time on dialysis), body surface area (BSA), body mass index (BMI), diabetes mellitus (DM), chronic heart failure (CHF), hepatitis $\mathrm{C}$ antibody, catheter $\mathrm{HD}$ access, cancer other than skin, and treatments per week. In addition, the following laboratory and treatment parameters were collected from electronic medical record (EMR): albumin, neutrophil-lymphocyte ratio (NLR), normalized protein catabolic rate (nPCR), dialysis adequacy (eKt/V), white blood cell count (WBC), and hemoglobin (HGB).

\section{Statistical Analysis}

Continuous variables were presented as mean \pm SD for normally distributed data or median and interquartile range for skewed data. Categorical variables were expressed as counts and percentages (\%). The $\chi^{2}$ test was performed on categorical data (e.g., gender), and $t$ tests were performed on continuous data (e.g., age). A logistic regression model was used to delineate the individual and combined effects of the measured parameters on vaccination response. A univariate logistic regression was performed for all relevant demographic, clinical, laboratory, and treatment related parameters. Multivariate regression models were created in an incremental approach examining the effect of dialysis start time and other a priori predictors of vaccination response. Models $1 \mathrm{~A}$ and $1 \mathrm{~B}$ examined dialysis start time and gender or age, respectively, as predictors of HB vaccination response. Model 2 examined the effects of dialysis start time, age, and gender on HB seroconversio. Models 3 and 4 were constructed with all the parameters from model 2 plus NLR, NLR and vintage, respectively. The order of including parameters in multivariate models was determined in the order of clinical significance of each parameter on vaccination response. All statistical analyses were performed using SAS 9.4.

\section{Results}

19,969 patients fit the inclusion criteria and were included in the analysis (Fig. 1). On average, the study cohort was $64.90 \pm 13.96$ years old, had a BMI of $29.54 \pm$ $9.69 \mathrm{~kg} / \mathrm{m}^{2}$, and was on dialysis for $0.86 \pm 1.61$ years, with hemoglobin levels of $11.22 \pm 0.77 \mathrm{~g} / \mathrm{dL}$ and $\mathrm{eKt} / \mathrm{V}$ of $1.55 \pm 0.29$. The study cohort was $58 \%$ male, $69 \%$ white, 
Fig. 2. Hepatitis $B$ vaccination response

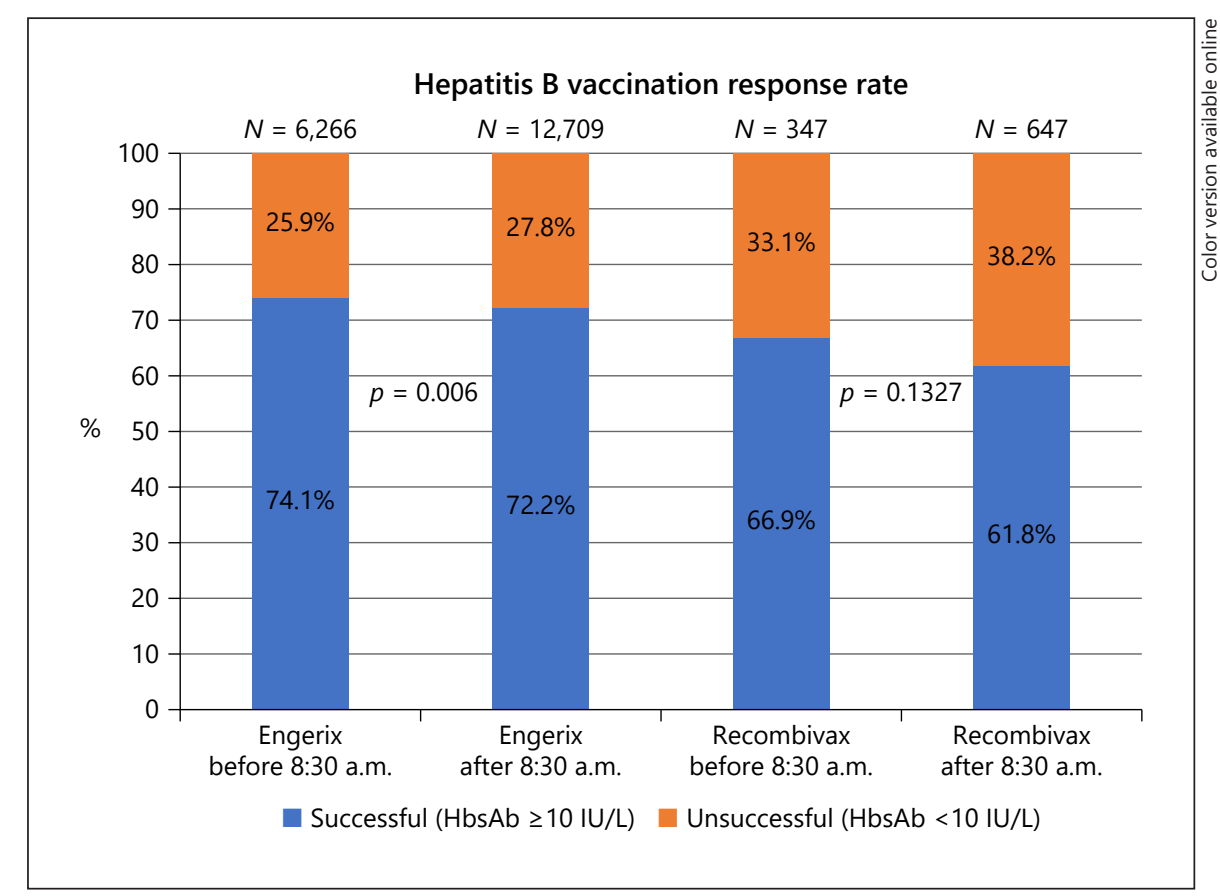

and $61 \%$ diabetic. $95 \%$ of the patients had received Engerix $B^{\circledR}$. Patients who received HD before 8:30 a.m. had significantly higher serum albumin, nPCR, hemoglobin levels, BMI, BSA, and dialysis vintage. Patients in this group also had significantly lower levels of NLR and WBC. In addition, patients treated before 8:30 a.m. were younger, more likely to be male, more likely to be hepatitis $\mathrm{C}$ positive, less likely to have central-venous catheter as vascular access, less likely to be white, and less likely to have CHF (Table 1). Patients who received Engerix $B^{\circledR}$ and Recombivax $\mathrm{HB}^{\mathrm{TM}}$ had a vaccination response rate of 73 and $64 \%$, respectively (Table 1). Patients who were vaccinated with Engerix $B^{\circledR}$ that started HD before 8:30 a.m. had a statistically significant higher rate of successful vaccinations compared to those who started HD treatments after 8:30 a.m. (Fig. 2).

In the univariate analysis, all the collected parameters were statistically significant predictors of vaccination success except for eKt/V and hepatitis $\mathrm{C}$ status. Vaccination with the Engerix $\mathrm{B}^{\circledR}$ vaccine protocol and receiving $90 \%$ of HD treatments starting before 8:30 a.m. were associated with higher odds of seroconversion. Higher levels of albumin, BSA, and nPCR increased the odds of successful vaccination, while higher NLR and WBC decreased the odds of successful vaccination. In addition, being younger, male, nonwhite, and on dialysis longer were associated with successful $\mathrm{HB}$ vaccination. Having a catheter as vascular access for $\mathrm{HD}$, presence of $\mathrm{CHF}$, and cancer other than skin were associated with lower odds to successful vaccination. Surprisingly, higher HGB levels were associated with lower odds of vaccination response, while prevalence of diabetes was associated with higher odds of vaccination response (Table 2).

The results from multivariate regression models demonstrated that dialysis start time was a significant predictor of $\mathrm{HB}$ vaccination response in model $1 \mathrm{~A}$ which included gender being male. In model $1 \mathrm{~B}$ to model 4 , the significance of starting dialysis before 8:30 a.m. as a predictor of $\mathrm{HD}$ vaccination response vanished, while age, gender (male vs. female), NLR, and vintage were all significant predictors (Table 3 ).

\section{Discussion}

In univariate regression, early dialysis start time (before 8:30 a.m.) is associated with higher odds of seroconversion. This association between early dialysis start time and vaccination response is no longer present in the multivariate regression which include potential confounders such as age, gender, NLR, and dialysis vintage.

There were marked differences between the early versus late cohorts which could account for the greater odds for $\mathrm{HB}$ vaccination response in the early group compared to the late group per the univariate analysis. $\mathrm{Pa}$ tients in the later group were on average older and less 
Table 2. Univariate models of hepatitis B vaccination response

\begin{tabular}{llllr}
\hline Parameter & Odds ratio & $\begin{array}{l}\text { Lower limit } \\
(95 \% \mathrm{CI})\end{array}$ & $\begin{array}{l}\text { Upper limit } \\
(95 \% \mathrm{CI})\end{array}$ & $p$ value \\
\hline Vaccine type: Engerix B & & 1.387 & 1.749 & $<0.0001$ \\
Albumin (g/L) & 1.557 & 2.287 & 2.666 & $<0.0001$ \\
Age (years) & 2.469 & 0.971 & 0.975 & $<0.0001$ \\
Race: white & 0.973 & 0.609 & 0.690 & $<0.0001$ \\
Body surface area (m $\left.{ }^{2}\right)$ & 0.648 & 0.738 & 0.918 & 0.0005 \\
Hemoglobin (g/dL) & 0.823 & 0.896 & 0.960 & $<0.0001$ \\
Sex: male & 0.928 & 1.086 & 1.214 & $<0.0001$ \\
Square root of HD vintage & 1.148 & 1.089 & 1.204 & $<0.0001$ \\
Hepatitis C positive & 1.145 & 0.894 & 1.157 & 0.8006 \\
Diabetes mellitus & 1.017 & 1.053 & 1.178 & 0.0002 \\
Neutrophil-to-lymphocyte ratio & 1.113 & 0.915 & 0.939 & $<0.0001$ \\
nPCR (g/kg/day) & 0.927 & 1.410 & 1.833 & $<0.0001$ \\
eKT/V & 1.607 & 0.942 & 1.135 & 0.4829 \\
WBC $(1,000 / \mu L)$ & 1.034 & 0.950 & 0.974 & $<0.0001$ \\
Vascular access: catheter & 0.962 & 0.837 & 0.962 & 0.0023 \\
CHF & 0.897 & 0.515 & 0.621 & $<0.0001$ \\
Cancer (other than skin) & 0.566 & 0.402 & 0.594 & $<0.0001$ \\
90\% of treatments starting before 8:30 a.m. & 0.488 & 1.107 & 1.036 & 0.0027 \\
\hline
\end{tabular}

nPCR, normalized protein catabolic rate; BMI, body mass index; WBC, white blood cells; CHF, chronic heart failure; $\mathrm{eKT} / \mathrm{V}$, dialysis adequacy.

Table 3. Multivariate models of hepatitis B vaccination response

\begin{tabular}{|c|c|c|c|c|}
\hline & Odds ratio & $\begin{array}{l}\text { Lower limit } \\
(95 \% \mathrm{CI})\end{array}$ & $\begin{array}{l}\text { Upper limit } \\
(95 \% \mathrm{CI})\end{array}$ & $p$ value \\
\hline \multicolumn{5}{|l|}{ Model 1A } \\
\hline $90 \%$ of treatments starting before $8: 30$ a.m. & 1.093 & 1.023 & 1.169 & 0.0089 \\
\hline Male versus female & 1.191 & 1.118 & 1.268 & $<0.0001$ \\
\hline \multicolumn{5}{|l|}{ Model 1B } \\
\hline $90 \%$ of treatments starting before $8: 30$ a.m. & 1.02 & 0.953 & 1.092 & 0.5643 \\
\hline Age & 0.973 & 0.97 & 0.975 & $<0.0001$ \\
\hline \multicolumn{5}{|l|}{ Model 2} \\
\hline $90 \%$ of treatments starting before $8: 30$ a.m. & 1.013 & 0.946 & 1.084 & 0.7166 \\
\hline Age & 0.973 & 0.971 & 0.975 & $<0.0001$ \\
\hline Male versus female & 1.15 & 1.079 & 1.225 & $<0.0001$ \\
\hline \multicolumn{5}{|l|}{ Model 3} \\
\hline $90 \%$ of treatments starting before $8: 30$ a.m. & 1 & 0.915 & 1.092 & 0.9965 \\
\hline Age & 0.974 & 0.971 & 0.977 & $<0.0001$ \\
\hline Male versus female & 1.163 & 1.072 & 1.261 & 0.0003 \\
\hline NLR & 0.948 & 0.935 & 0.962 & $<0.0001$ \\
\hline \multicolumn{5}{|l|}{ Model 4} \\
\hline $90 \%$ of treatments starting before $8: 30$ a.m. & 0.98 & 0.891 & 1,079 & 0.6803 \\
\hline Age & 0.975 & 0.971 & 0.978 & $<0.0001$ \\
\hline Male versus female & 1.193 & 1.094 & 1.301 & $<0.0001$ \\
\hline NLR & 0.96 & 0.946 & 0.975 & $<0.0001$ \\
\hline Vintage & 1.17 & 1.086 & 1.259 & $<0.0001$ \\
\hline
\end{tabular}

NLR, neutrophil-to-lymphocyte ratio. 
likely to be male. It has been previously reported that younger age and female gender were positively associated with vaccination response [12]. In addition, patients in the early group had longer vintage which has been shown to be associated with higher vaccine response rates [13].

It has been shown that patients who are dialyzed in the early morning have on average decreased sleep duration in the night before HD compared to those who receive dialysis later in the day; during the night following dialysis, these patients slept significantly more than the night prior to treatment [11]. These patients receiving dialysis in the early morning shift also slept significantly longer compared to those patients in later shifts in the night after dialysis with differences ranging from 36 to $53 \mathrm{~min}$ [11]. Because of that sleep pattern, we hypothesized that the seroconversion rate might be higher in patients with an early dialysis start. Univariate analysis corroborated that hypothesis: we observed that an early dialysis start time was associated with a greater odd of vaccine response.

In our univariate models, the presence of DM was associated with greater odds of vaccination response. However, this contradicts previous findings which state that higher prevalence of DM is associated with lower chance of vaccination response [14]. Other reports found that there was no significant association between DM status and seroconversion in ESKD populations $[15,16]$. Also, interestingly, higher HGB levels were associated with decreased odds of vaccination response. In another study carried out in HD and peritoneal dialysis patients, higher HGB levels were found to be associated with greater odds of vaccination response in univariate regression [17].

A major shortcoming is the lack of measured sleep time in our study cohort. We used dialysis start time as a surrogate indicator of sleep duration in the night following HD. This approach was motivated by findings regarding postdialysis sleep duration made in an earlier study. To overcome the obvious weakness of the current study, future research should aim to investigate the association between patient-specific measured sleep time and seroconversion following HB vaccination. The advent of multiple pervasive sensing devices will allow for a large-scale exploration of that question.

In summary, in univariate analysis, a start of HD prior to 8:30 a.m. - a surrogate indicator of longer sleeping time in the night following vaccination - is associated with a higher seroconversion rate following hepatitis $B$ vaccination. This finding corroborates observations in healthy subjects in whom there is an association between longer postvaccination sleep duration and higher seroconversion rate $[3,4]$. In our study population, this association was no longer present after multivariate adjustments. Research is warranted to explore the association between measured postvaccination sleep duration and seroconversion rate.

\section{Statement of Ethics}

This study was conducted in accordance with the Declaration of Helsinki. Western Institutional Review Board's (WIRB's) IRB Affairs Department reviewed the exemption criteria under 45 CFR $\$ 46.101$ (b)(4), which states that the following category of research is exempt from the requirements of 45 CFR 46: "research, involving the collection or study of existing data, documents, records, pathological specimens, if these sources are publicly available or if the information is recorded by the investigator in such a manner that subjects cannot be identified, directly or through identifiers linked to the subjects." This project meets the conditions for exemption under 45 CFR 46.101(b)(4). All of the data are in existence as of December 31,2015, and the information will be recorded in such a manner that subjects cannot be identified, directly or through identifiers linked to the subjects. It was also confirmed that the results of this study will not be submitted to the Food and Drug Administration (FDA) for marketing approval.

\section{Conflict of Interest Statement}

M.H., X.Y., S.T., and P.K. are employees of Renal Research Institute, a wholly owned subsidiary of Fresenius Medical Care North America. J.H. is an employee of Fresenius Medical Care North America. F.W.M. is an employee of Fresenius Medical Care Management AG. F.W.M., J.H., P.K., and S.T. hold performance shares in Fresenius Medical Care. P.K. receives author honorarium from UpToDate.

\section{Funding Sources}

This study was fully funded by Renal Research Institute.

\section{Author Contributions}

M.H., X.Y., S.R., S.W., S.T., J.H., F.W.M., and P.K. were responsible for study design. X.Y. was responsible for data extraction and analysis. M.H., X.Y., S.R., S.W., S.T., J.H., F.W.M., and P.K. were responsible for interpretation of the study results. M.H., X.Y., S.T., and P.K. were responsible for the preparation of the manuscript, tables, and figures. All authors reviewed the manuscript prior to submission. 


\section{References}

1 Kato S, Chmielewski M, Honda H, PecoitsFilho R, Matsuo S, Yuzawa Y, et al. Aspects of immune dysfunction in end-stage renal disease. Clin J Am Soc Nephrol. 2008;3(5):152633.

2 Kim KW, Chung BH, Jeon EJ, Kim BM, Choi BS, Park CW, et al. B cell-associated immune profiles in patients with end-stage renal disease (ESRD). Exp Mol Med. 2012 Aug 1;44(8): 465-72.

3 Lange T, Perras B, Fehm HL, Born J. Sleep enhances the human antibody response to hepatitis A vaccination. Psychosom Med. 2003 Sep-Oct;65(5):831-5.

4 Prather AA, Hall M, Fury JM, Ross DC, Muldoon MF, Cohen S, et al. Sleep and antibody response to hepatitis $\mathrm{B}$ vaccination. Sleep. 2012 Aug;35(8):1063-9.

5 Spiegel K, Sheridan JF, Van Cauter E. Effect of sleep deprivation on response to immunization. JAMA. 2002 Sep 25;288(12):1471-2.

6 Cohen S, Doyle WJ, Alper CM, Janicki-Deverts D, Turner RB. Sleep habits and susceptibility to the common cold. Arch Intern Med. 2009 Jan 12;169(1):62-7.
7 Lange T, Dimitrov S, Bollinger T, Diekelmann S, Born J. Sleep after vaccination boosts immunological memory. J Immunol. 2011 Jul 1;187(1):283-90.

8 Irwin MR, Olmstead R, Carroll JE. Sleep disturbance, sleep duration, and inflammation: a systematic review and meta-analysis of cohort studies and experimental sleep deprivation. Biol Psychiatry. 2016 Jul 1;80(1):40-52.

9 Iliescu EA, Yeates KE, Holland DC. Quality of sleep in patients with chronic kidney disease. Nephrol Dial Transplant. 2004 Jan;19(1):959.

10 Parker KP, Bliwise DL, Bailey JL, Rye DB. Polysomnographic measures of nocturnal sleep in patients on chronic, intermittent daytime haemodialysis vs those with chronic kidney disease. Nephrol Dial Transplant. 2005 Jul;20(7):1422-8.

11 Han M, Williams S, Mendoza M, Ye X, Zhang $\mathrm{H}$, Calice-Silva V, et al. Quantifying physical activity levels and sleep in hemodialysis patients using a commercially available activity tracker. Blood Purif. 2016;41(1-3):194-204.

12 Nahar K, Jahan M, Nessa A, Tabassum S. Antibody responses after hepatitis B vaccination among maintenance haemodialysis patients. Bangladesh Med Res Counc Bull. 2011 Dec; 37(3):88-91.
13 Steketee RW, Ziarnik ME, Davis JP. Seroresponse to hepatitis B vaccine in patients and staff of renal dialysis centers, Wisconsin. Am J Epidemiol. 1988 Apr;127(4):772.

14 Taheri S, Shahidi S, Moghtaderi J, Seirafian S, Emami A, Eftekhari S. Response rate to hepatitis $B$ vaccination in patients with chronic renal failure and end-stage-renal-disease: influence of diabetes mellitus. J Res Med Sci. 2005; 10(6):384-90.

15 Fernández E, Betriu MA, Gómez R, Montoliu $\mathrm{J}$. Response to the hepatitis $\mathrm{B}$ virus vaccine in haemodialysis patients: influence of malnutrition and its importance as a risk factor for morbidity and mortality. Nephrol Dial Transplant. 1996;11(8):1559-63.

16 Sezer S, Ozdemir FN, Güz G, Arat Z, Colak T, Sengul S, et al. Factors influencing response to hepatitis $\mathrm{B}$ virus vaccination in hemodialysis patients. Transplant Proc. 2000 May;32(3): 607-8.

17 Lacson E, Teng M, Ong J, Vienneau L, Ofsthun N, Lazarus JM. Antibody response to Engerix-B and Recombivax-HB hepatitis $\mathrm{B}$ vaccination in end-stage renal disease. Hemodial Int. 2005 Oct;9(4):367-75. 